\title{
Managing the Bright and Dark Sides of Humorous Response in Service Recovery: An Abstract
}

\author{
Hyunju Shin and Lindsay R. L. Larson
}

\begin{abstract}
Despite humor's promising ability to positively address customer complaints, little research has sought to investigate its role in recovering from service failure on social media. To address this research gap, this study investigates the role of humorous response to customer complaints on social media. We postulate that humorous response has a favorable influence on perceived excitement of the firm. However, humorous response needs to be implemented with care as it negatively influences perceived trustworthiness of as well as overall attitude toward the firm. However, when the customer complaint is also humorous, a humorous response is more effective in intensifying the positive effect on perceived excitement of the firm and lowering its negative effect on perceived trustworthiness of and overall attitude toward the firm. The current study utilizes a scenario-based experiment in the restaurant context. The findings of this study will provide insights into how service organizations can better utilize the humorous response strategy in response to customer complaints on social media.
\end{abstract}

References Available Upon Request

H. Shin $(\bowtie) \cdot$ L. R. L. Larson

Georgia Southern University, Statesboro, GA, USA

e-mail: hshin@georgiasouthern.edu; lindsaylarson@georgiasouthern.edu 